Tropical Journal of Pharmaceutical Research June 2015; 14 (6): 1005-1011

ISSN: $1596-5996$ (print); 1596-9827 (electronic)

(C) Pharmacotherapy Group, Faculty of Pharmacy, University of Benin, Benin City, 300001 Nigeria.

All rights reserved.

Available online at http://www.tjpr.org

Original Research Article

http://dx.doi.org/10.4314/tjpr.v14i6.10

\title{
Withaferin A Suppresses Liver Tumor Growth in a Nude Mouse Model by Downregulation of Cell Signaling Pathway Leading to Invasion and Angiogenesis
}

\author{
Yu-Xu Wang*, Wei-Bao Ding and Cheng-Wei Dong \\ Department of Hepatobiliary Surgery, Weifang People's Hospital, Weifang 261041, China
}

*For correspondence: Email: wangyuxu88@gmail.com; Tel/Fax: 0086-536-8234981

Received: 23 November 2014

Revised accepted: 19 May 2015

\begin{abstract}
Purpose: To investigate the effect of withaferin A on tumor growth and metastasis in liver in a nude mouse model.

Methods: Withaferin A was injected through a portal vein to the orthotopic liver tumor in a nude mice model. Xenogen in vivo imaging system was used to monitor tumor growth and metastasis. The effect of withaferin A on tumor volume, invasive growth pattern, expression of Pyk2, upregulation of BAX/P53, apoptotic signaling and ROCKIP10/VEGF pathway along with cytoskeletal protein actin projection formation was studied. Tumor/non-tumor margin was examined under electron microscopy. In addition, the direct effect of withaferin A on liver cancer cells and endothelial cells was further investigated.

Results: A significant inhibition of tumor growth and lower incidence of lung metastasis was observed after withaferin $A$ treatment. Withaferin A treatment led to a decrease in the incidence of intrahepatic metastasis from 90 (9 of 10) to $10 \%$ (1 of 10, $p=0.041$ ). There was decrease in macrophage infiltration in the liver tumors and vessels. Western blot analysis revealed inhibition of expression of Pyk2, ROCK1 protein and VEGF. Electron microscopy showed tumor vascular endothelial cell damage and significant necrosis of tumor tissues. It also suppressed formation of cytoskeletal protein actin projection involved in cell migration.

Conclusion: Withaferin A inhibits liver tumor invasion and angiogenesis by downregulation of cell signalling pathway leading to invasion and angiogenesis. Therefore, withaferin $A$ is a promising candidate for the treatment of liver tumor invasion and angiogenesis.
\end{abstract}

Keywords: Withaferin A, Macrophage, Lung metastasis, Angiogenesis, Vascular endothelial growth factor, Rho kinase, Withania somnifera

Tropical Journal of Pharmaceutical Research is indexed by Science Citation Index (SciSearch), Scopus, International Pharmaceutical Abstract, Chemical Abstracts, Embase, Index Copernicus, EBSCO, African Index Medicus, JournalSeek, Journal Citation Reports/Science Edition, Directory of Open Access Journals (DOAJ), African Journal Online, Bioline International, Open-J-Gate and Pharmacy Abstracts

\section{INTRODUCTION}

Due to difficulty in detection, aggressive and chemoresistant behaviour of metastatic cells no efficient treatments are available for metastatic disease [1]. Invasion of tumor cells into surrounding tissue, intravasation into blood or lymphatic vessels and extravasation into new host environment are triggered by a process termed as epithelial mesenchymal transition
(EMT) $[2,3]$. It is reported that cancer cells can re-trigger EMT to migrate into surrounding tissue $[3,4]$. Rho kinase (ROCK) plays a significant role in regulating cytoskeletal events and invasion of hepatocellular carcinoma $[5,6]$. It is believed that IFN-inducible protein 10 (IP10) which promotes tumor cell invasiveness in colon cancer [7] and is related to angiogenesis in uterine cervical cancers [8] has key role in hepatocellular carcinoma invasiveness. Vascular endothelial 
growth factor (VEGF) and matrix metalloproteinase 9 (MMP9) secreted by macrophages promote tumor invasion and angiogenesis $[9,10]$. Angiopoietins in the presence of VEGF may promote tumor angiogenesis and progression in human hepatocellular carcinoma [11].

Withaferin A (1), a steroidal lactone is isolated from Withania somnifera, the extracts of which are used in traditional East Indian medicine [12]. Out of 14 withanolides isolated from $W$. somnifera, withaferin $A$ is present as a major compound [13,14]. Withaferin $A$ has antiangiogenic effect $[12,15]$ at micromolar doses and inhibits soft tissue sarcoma growth and local recurrence in xenograft experiments [16]. It is reported to have proapoptotic and anti-tumor activity in breast and prostate cancers [17-19]. Withaferin $A$ acts on NF-Kb, BCL-2, FOXO3A, Hsp90, phosphorylated STAT3 and annexin II [18,20-24].

Taking cue from the literature, we designed an experiment to study the effect of withaferin $A$ on suppression of liver tumor growth in a nude mouse model with an attempt to develop a potent therapeutic agent.

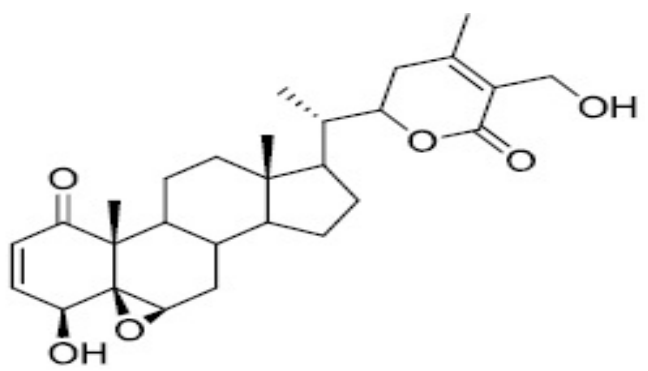

Fig 1: Structure of withaferin A

\section{EXPERIMENTAL}

\section{Cell lines}

Human hepatocellular carcinoma cell lines MHCC97 and $\mathrm{JHH}-5$ were maintained in DMEM with high glucose (Life Technologies) supplemented with $10 \%$ heat-inactivated fetal bovine serum (Life Technologies), $100 \mathrm{mg} / \mathrm{mL}$ penicillin $\mathrm{G}$, and $50 \mu \mathrm{g} / \mathrm{mL}$ streptomycin (Life Technologies) at $37{ }^{\circ} \mathrm{C}$ in a humidified atmosphere containing $5 \% \mathrm{CO}_{2}$.

\section{Orthotopic nude mice liver tumor model}

MHCC97 cells $\left(6 \times 10^{5}\right)$ were cultured in culture medium and harvested. After cell viability was determined, $2 \times 10^{6}$ viable cells were injected subcutaneously at the proximal dorsal midline of 6-week-old male athymic nu/nu mice (Harlan). The tumors were removed after attaining 0.8 to 1 $\mathrm{cm}$ diameter, cut into cubes of 1 to $2 \mathrm{~mm}^{3}$ in size, which were then implanted into the left liver lobes of another group of nude mice. The study was approved by the institutional committee for animal care and use.

\section{Treatment, imaging, and sample collection}

Withaferin $A$ (withaferin $A$, treatment group) or phosphate-buffered saline (PBS) solution (control group) was injected via the portal vein after tumor implantation. Blood samples were collected every week from the tail vein for the detection of serum levels of withaferin A. Mice bearing liver tumors were checked by a higherresolution $7 \mathrm{~T}$ magnetic resonance imaging scanner, PharmaScan 70/16 (Bruker BioSpin). Liver tumor growth and lung metastasis were monitored by Xenogen IVIS every week. The nude mice were sacrificed at weeks 4,5 , and 6 , respectively, after tumor implantation. Liver, lung, kidney, and spleen suspected of tumor metastasis were sampled for tissue sectioning to examine intrahepatic, lung, kidney, and spleen metastases.

\section{Light and transmission electron microscopy}

Tumor tissues in liver were collected for light microscopy with H\&E staining at different time points after tumor implantation. The specimens were immediately cut into $1-\mathrm{mm}$ cubes and fixed in $2.5 \%$ glutaraldehyde in sodium carcodylate hydrochloride buffer overnight at $4{ }^{\circ} \mathrm{C}$ for electron microscopy section. The sections were then examined under a transmission electron microscope, Philips EM 208 (Koninklijke Philips Electronics, Eindhoven, Netherlands).

\section{Gene expression of ROCK1, IP10, and MMP9}

Total RNA $(0.5 \mu \mathrm{g})$ isolated from liver tumor cells using RNeasy Midi kit (Qiagen) was subjected to reverse transcription reaction using TaqMan Reverse Transcription Reagents (ref. 24; Applied Biosystems). The product $(1 \mu \mathrm{L})$ was used to perform real-time quantitative reverse transcription-PCR using TaqMan Core Reagent kit (Applied Biosystems). The ABI PRISM 7900HT Sequence Detection System (PE Applied Biosystems) was used for real-time cDNA quantitation of ROCK, IP10, angiopoietin 1 , and VEGF.

The primer sets and probes were also purchased from Applied Biosystems. Human GAPDH was used as an endogenous control (TaqMan human 
GAPDH Control Reagents kit, Applied Biosystems). The $2^{-\Delta \Delta C t}$ method was used to calculate the relative fold difference of ROCK, IP10, angiopoietin 1, and VEGF expressions in all samples.

\section{Western blot analysis}

Proteins were isolated with IP buffer, and total protein concentration was determined using the Bio-Rad (Hercules, CA) assay. Total protein (50 $\mu \mathrm{g})$ were loaded per well. Blotted membranes were incubated with primary antibody. AntiROCK-1, anti-0IP10, and anti-VEGF antibodies were purchased from BD Biosciences, San Jose, CA. Anti-Akt, anti-phospho-Akt (Ser473), antiBcl-2, anti-BAX, and anti-phospho-p53 (Ser15) antibodies were from Cell Signaling Technology.

\section{Liquefied matrigel assay for tube formation}

MHCC97 cells $\left(1 \times 10^{5}\right)$ were seeded in Liquefied Matrigel (BD Biosciences) precoated 96-well plate. Withaferin $A$ was added to the wells at a dose of $50 \mu \mathrm{g} / \mathrm{mL}$. Digital camera was used to record the appearance of the cells on Matrigel.

\section{Statistical analysis}

All data represent the mean of three independent experiments. The significance of differences between means was determined by Student's ttest. $P<0.05$ was considered statistically significant.

\section{RESULTS}

\section{Effect of withaferin A on liver tumor growth and metastasis}

To determine the effect of withaferin A treatment on tumor volume, orthotopic tumor implantation was followed by measurement of tumor volume after 4, 5, and 6 weeks (Fig. 2A and B; Table 1). A decrease in incidence of intrahepatic metastasis was observed from $90 \%$ (9 of 10) to $10 \%$ after withaferin $A$ treatment. There was also suppression in lung metastasis on treatment with withaferin $A$. Incidence of lung metastasis in mice decreased from 80 (8 of 10) to $10 \%$ (1 of 10).

Table 1: Comparison of tumor volume from mice with or without withaferin $A$ treatment

\begin{tabular}{lccc}
\hline Duration after tumor implantation & $\begin{array}{c}\text { Treatment } \\
(\mathbf{n}=\mathbf{1 0})\end{array}$ & $\begin{array}{c}\text { Control } \\
(\mathbf{n}=\mathbf{1 0})\end{array}$ & $\boldsymbol{P}$-value \\
\hline 4 wk, volume of tumor $\left(\mathrm{mm}^{3}\right)$ & 16.87 & 1122 & 0.005 \\
5 wk, tumor volume $\left(\mathrm{mm}^{3}\right)$ & 72.33 & 1,267 & 0.004 \\
6 wk, tumor volume $\left(\mathrm{mm}^{3}\right)$ & 62 & 2,640 & 0.004 \\
\hline
\end{tabular}

Note: Median (range)

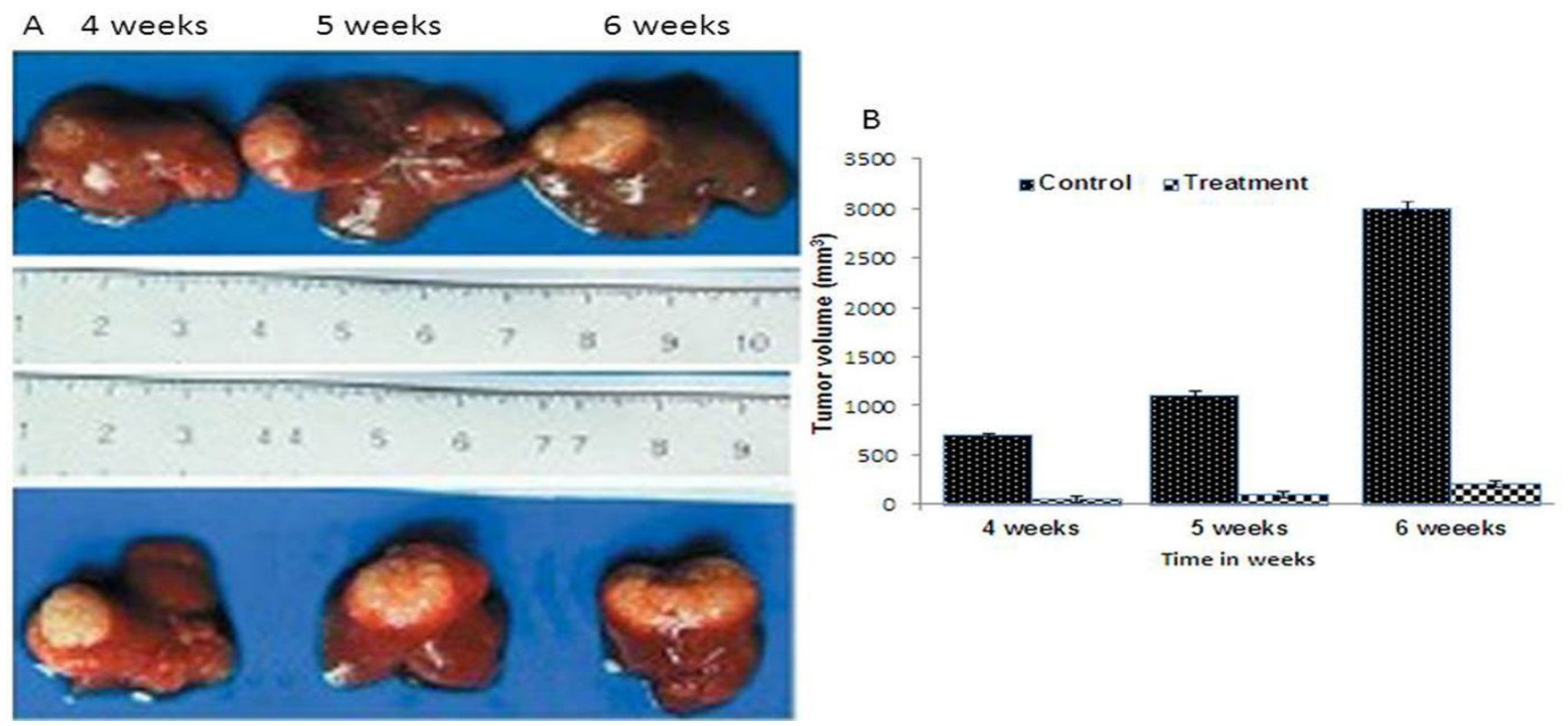

Fig. 2: The effect of withaferin A on in vivo tumor growth and metastasis of HCC. Withaferin A treatment suppressed tumor growth and metastasis in subcutaneous and orthotopic implantation mouse models. Metastatic lesions in the lungs of the orthotopic implantation models at indicated time points 


\section{Effect of withaferin A on invasive growth pattern of tumor}

Withaferin A treatment leads to significant necrosis of tumor tissues in treatment group compared to that of control group. In control group, we observed tumor cell migration and invasion of foreign tissue space using light microscopic examination (Fig 3). Withaferin A treatment leads to ultrastructural changes in liver tumor tissue and non-tumor tissue. Intrahepatic metastasis, presence of intact tumor cells and invasion of tumor cells to blood vessels were obvious in control group. On the other hand, there was clear separation between tumor cells and liver cells and tumor endothelial cell disruption in the mice treated with withaferin $A$. The disruption of tumor endothelial cells might have contributed to inhibition of tumor angiogenesis.

\section{Effect of withaferin A on Pyk2 expression and tumor cell apoptosis}

The effect of withaferin $A$ treatment on expression of the intracellular protein, Pyk2, in liver tumors was investigated. Western blot analysis demonstrated that withaferin A exposure significantly decreased the expression of Pyk2 in comparison to that in control group (Fig 4A). The decrease was significant at $30 \mu \mathrm{g}$ of withaferin $A$ treatment after 5 weeks. There was also apoptosis induction in tumor cells after treatment with withaferin $A$ compared to untreated cells (Fig. 4B). An intracellular protein, Pyk2 is involved in the invasion of liver tumor and hindrance to treatment [25]. Pyk2 activates cSrc/extracellular signal-regulated kinase which enhances cell proliferation and invasiveness of hepatocellular carcinoma [26,27].
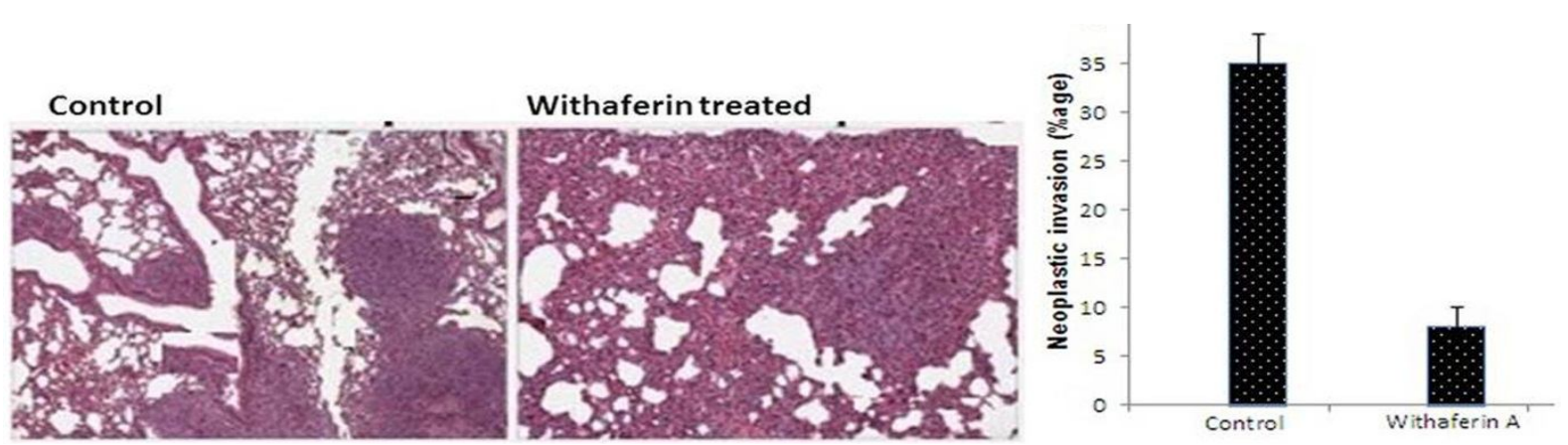

Fig 3: Tumor cell migration and invasion of foreign tissue space using light microscopic examination

A

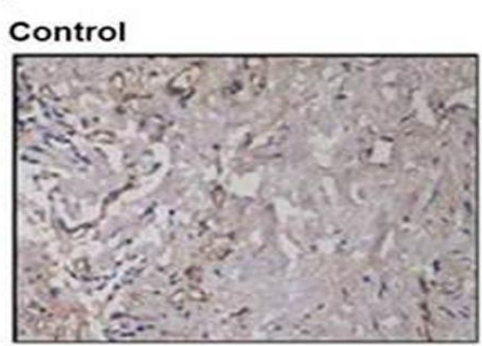

B
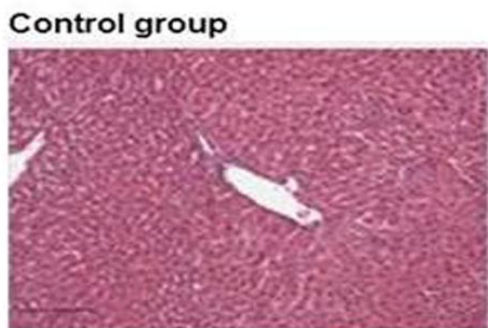

Withaferin A

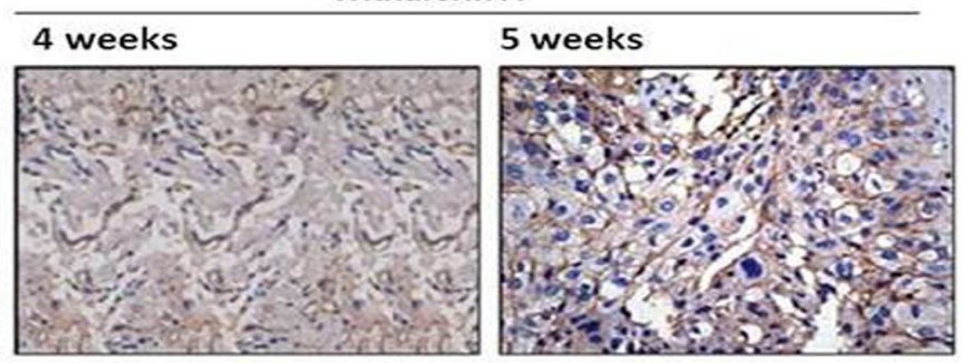

4 weeks

Withaferin A treated group

\section{6 weeks}
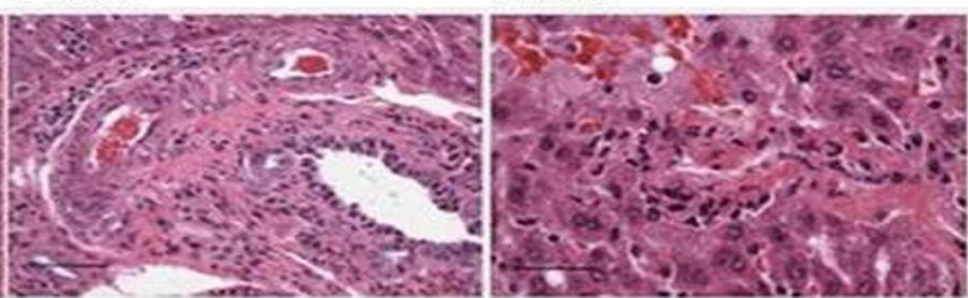

Fig 4: (A) Intracellular protein expression of Pyk2 in liver tumor was examined by immunostaining. (B) tumor cell apoptosis was compared by terminal deoxynucleotidyl transferase-mediated dUTP nick end labeling staining. Comparison of apoptotic tumor cells of mice with and without treatment at different time points 
Effect of withaferin A on apoptotic signaling and ROCK/IP10/VEGF pathway in liver cancer cells and inhibition of tube formation of tumor endothelial cells

The effect of withaferin $A$ on apoptotic signalling of $\mathrm{JHH}-5$ and $\mathrm{MHCC} 97$ cells were investigated by Western blot (Fig 5A). The down regulation of Akt signal and up regulation of BAX/P53 was mainly found in $\mathrm{JHH}-5$ cell line. Withaferin $A$ administration down-regulated the protein expression of ROCK, IP10, and VEGF in MHCC97 liver tumor cells in a dose-dependent manner (Fig 5B). Withaferin A also inhibited the tube formation ability of mouse endothelial cells (cell line CRL2279) in tube formation assay (Fig 5C).

\section{Effect of withaferin A treatment on cytoskeletal protein actin projection formation}

The MHCC97 liver tumor cells were observed to give rise to obvious cytoskeletal protein actin projection formation (Fig 6). However, exposure of the MHCC97L liver tumor cells to $50 \mu \mathrm{M}$ concentration of withaferin A suppressed the formation of cytoskeletal protein actin projection (Fig 6).

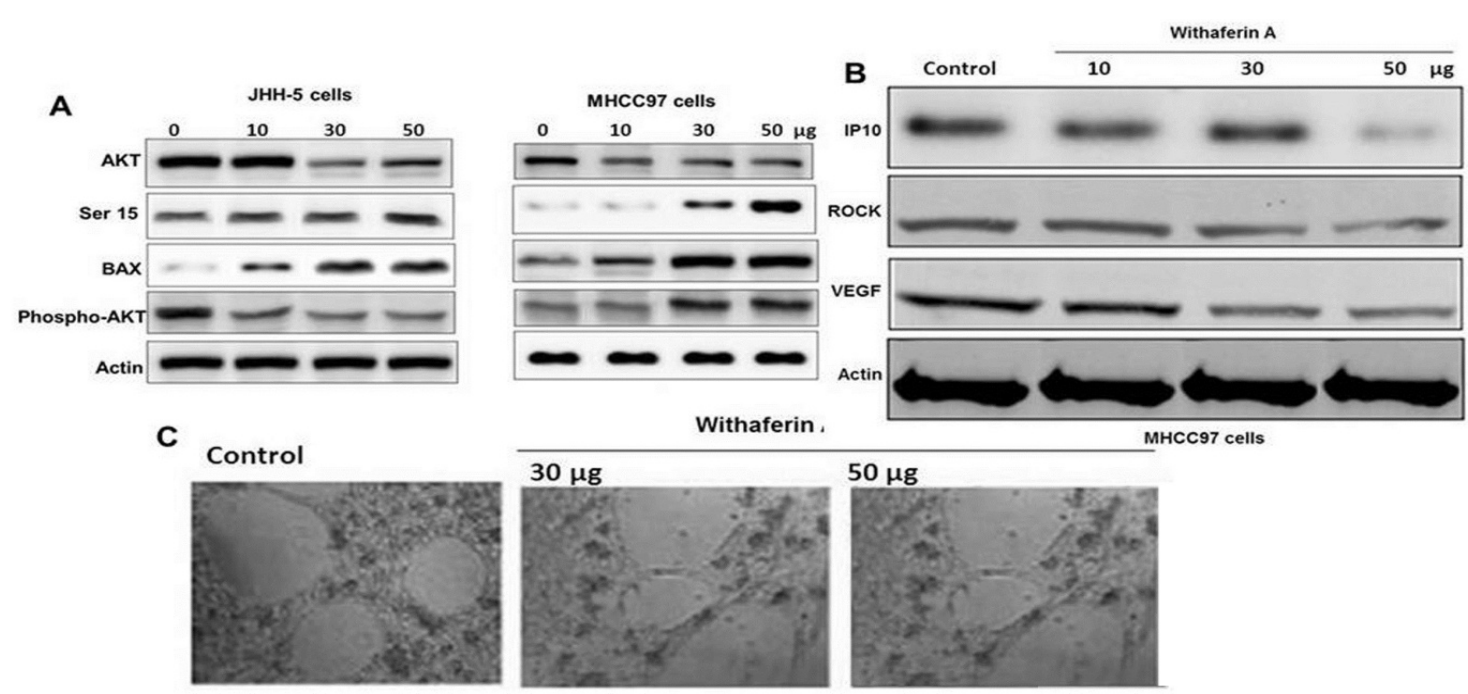

Fig 5: Western blot analysis. (A) Expression of Akt and BAX/P53 under withaferin A treatment was studied. (B) Protein expression of ROCK, IP10, and VEGF was compared in MHCC97L cells under withaferin A treatment. (C) Comparison of tube formation in mouse endothelial cells with or without withaferin $A$ administration
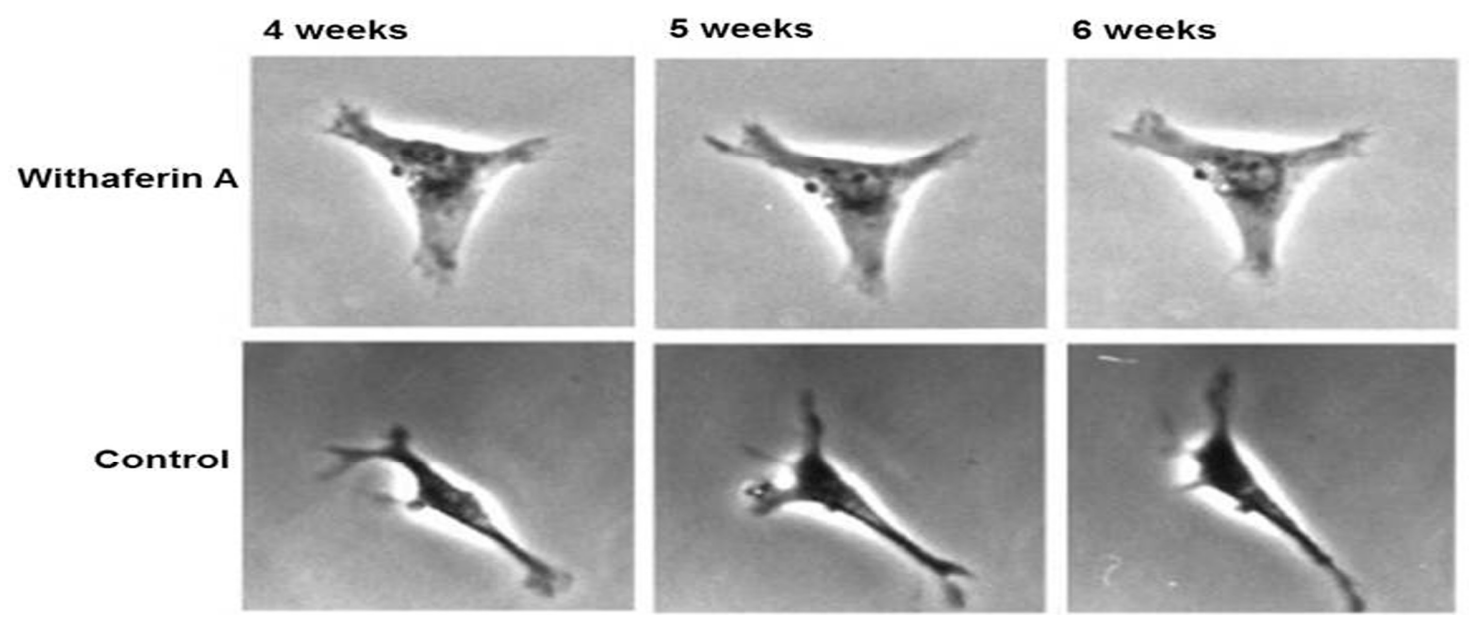

Fig 6: Comparison of cytoskeletal protein actin projection formation on tumor cell surface. Without withaferin $A$ treatment, obvious cytoskeletal protein actin projection formation was found on MHCC97L liver tumor cell surface. After administration of withaferin $A$ at concentrations of $50 \mu \mathrm{g}$, only a few cytoskeletal protein actin projection were formed on the tumor cell surface 


\section{DISCUSSION}

The study demonstrated the effect of withaferin A in tumor growth and metastasis inhibition in an orthotopic liver tumor model. There was significant suppression of liver tumor growth by withaferin A treatment after different intervals of time shown by using Xenogen IVIS and higherresolution micro-magnetic resonance imaging. Withaferin A treatment also decreased the incidence of local and distant metastasis. The histological examination of control group showed presence of invasive tumor growth in the form of tumor thrombus and intrahepatic metastasis. However, withaferin A treated group showed tumor necrosis and clear demarcation between tumor and non-tumor tissues. Withaferin $A$ treatment damaged the tumor endothelial cells and lead to suppression of tumor angiogenesis. In the control group, the electron microscopic examination showed long sinusoids and tumor cell invasion into lymphatic and blood vessels. The formation of sinusoids and tumor cell invasion into vessels clearly indicate tumor angiogenesis and invasiveness.

To investigate the mechanism behind inhibition of tumor growth and angiogenesis by withaferin A, we monitored the expression of liver tumor genes IP10/ROCK/Pyk2. The western blot analysis revealed that withaferin $A$ treatment decreased the expression of Pyk2, an intracellular protein involved in the invasion of liver tumor and hindrance to treatment [25]. Withaferin A also inhibited tumor-associated macrophage infiltration in liver tumors. Tumor invasion and angiogenesis is mediated by macrophages [9] by secreting inflammatory cytokines and chemokines (VEGF and MMP9) [10]. Withaferin $A$ treatment inhibited the expression of VEGF. Obviously, liver tumor growth and metastasis were significantly suppressed by withaferin A treatment.

The formation of cytoskeletal protein actin projection on cell surface leads to cell motility. Withaferin A treatment inhibited the formation of cytoskeletal protein actin projections on cell surface and intracellular protein levels of IP10/ROCK/VEGF in a dose-dependent manner. In addition, it inhibited formation of tubes in endothelial cells and induced apoptosis of tumor cells in the animal model.

\section{CONCLUSION}

Withaferin A inhibits liver tumor invasion and angiogenesis by downregulation of cell signaling pathway leading to invasion and angiogenesis. It also inhibits tumor-associated macrophage infiltration leading to tumor inflammatory response. Therefore, withaferin $A$ can be a promising candidate for the treatment of liver tumor invasion and angiogenesis.

\section{REFERENCES}

1. Stafford LJ, Vaidya KS, Welch DR. Metastasis suppressors genes in cancer. Int J Biochem Cell Biol 2008; 40: 874-891.

2. Lillie FR. The development of the chicked. New York: $H$. Holt and company, 1908.

3. Huber MA, Kraut $N$, Beug $H$. Molecular requirements for epithelial-mesenchymal transition during tumor progression. Curr Opin Cell Biol 2005; 17: 548-558.

4. Thiery JP. Epithelial-mesenchymal transitions in tumour progression. Nat Rev Cancer 2002; 2: 442-454.

5. Wong CC, Wong CM, Tung EK, Man K, Ng IO. Rhokinase 2 is frequently overexpressed in hepatocellular carcinoma and involved in tumor invasion. Hepatology 2009; 49: 1583-1594.

6. Wong CC, Wong CM, Ko FC, et al. Deleted in liver cancer 1 (DLC1) negatively regulates Rho/ROCK/MLC pathway in hepatocellular carcinoma. PLoS ONE 2008; 3: e2779.

7. Zipin-Roitman A, Meshel T, Sagi-Assif O. CXCL10 promotes invasion-related properties in human colorectal carcinoma cells. Cancer Res 2007; 67: 3396-3405.

8. Sato E, Fujimoto J, Toyoki $H$, et al. Expression of IP-10 related to angiogenesis in uterine cervical cancers. $\mathrm{Br}$ J Cancer 2007; 96: 1735-1739.

9. Condeelis J, Pollard JW. Macrophages: obligate partners for tumor cell migration, invasion, and metastasis. Cell 2006; 124: 263-266.

10. Coussens LM, Tinkle CL, Hanahan D, Werb Z. MMP-9 supplied by bone marrow-derived cells contributes to skin carcinogenesis. Cell 2000; 103: 481-490.

11. Mitsuhashi N, Shimizu H, Ohtsuka M, et al. Angiopoietins and Tie-2 expression in angiogenesis and proliferation of human hepatocellular carcinoma. Hepatology 2003; 37: 1105-1113.

12. Mohan R, Hammers HJ, Bargagna-Mohan P, Zhan XH, Herbstritt CJ, Ruiz A, Zhang L, Hanson AD, Conner $B P$, Rougas J, Pribluda VS. Withaferin $A$ is a potent inhibitor of angiogenesis. Angiogenesis 2004; 7: 115122.

13. Misra L, Mishra $P$, Pandey A, Sangwan RS, Sangwan NS, Tuli R. Withanolides from Withania somnifera roots. Phytochemistry 2008; 69: 1000-1004.

14. Chaurasiya ND, Uniyal GC, Lal P, Misra L, Sangwan NS, Tuli R, Sangwan RS. Analysis of withanolides in root and leaf of Withania somnifera by HPLC with photodiode array and evaporative light scattering detection. Phytochem Anal 2008; 19: 148-154.

15. Bargagna-Mohan $P$, Hamza A, Kim YE, Khuan Abby Ho Y, Mor-Vaknin N, Wendschlag N, Liu J, Evans RM, Markovitz DM, Zhan CG, Kim KB, Mohan R. The 
tumor inhibitor and antiangiogenic agent withaferin $A$ targets the intermediate filament protein vimentin. Chem Biol 2007; 14: 623-634.

16. Lahat G, Zhu QS, Huang KL, Wang S, Bolshakov S, Liu $J$, Torres K, Langley RR, Lazar AJ, Hung MC, Lev D. Vimentin is a novel anti-cancer therapeutic target; insights from in vitro and in vivo mice xenograft studies. PLoS One 2010; 5: e10105.

17. Shohat B, Gitter $S$, Abraham A, Lavie D. Antitumor activity of withaferin A (NSC-101088). Cancer Chemother Rep 1967; 51: 271-276.

18. Stan $S D$, Hahm ER, Warin R, Singh SV. Withaferin $A$ causes FOXОЗа- and Bimdependent apoptosis and inhibits growth of human breast cancer cells in vivo. Cancer Res 2008; 68: 7661-7669.

19. Srinivasan S, Ranga RS, Burikhanov R, Han SS, Chendil D. Par-4-dependent apoptosis by the dietary compound withaferin $A$ in prostate cancer cells. Cancer Res 2007; 67: 246-253.

20. Falsey RR, Marron MT, Gunaherath GM, Shirahatti $N$, Mahadevan D, Gunatilaka AA, Whitesell L. Actin microfilament aggregation induced by withaferin $A$ is mediated by annexin II. Nat Chem Biol 2006; 2: 3338.

21. Singh $D$, Aggarwal A, Maurya R, Naik S. Withania somnifera inhibits NF-kappaB and AP-1 transcription factors in human peripheral blood and synovial fluid mononuclear cells. Phytother Res 2007; 21: 905913.

22. Koduru S, Kumar R, Srinivasan S, Evers MB, Damodaran C. Notch-1 inhibition by Withaferin-A: a therapeutic target against colon carcinogenesis. Mol Cancer Ther 2010; 9: 202-210.

23. Panjamurthy K, Manoharan S, Nirmal MR, Vellaichamy L. Protective role of Withaferin-A on immunoexpression of $p 53$ and bcl-2 in 7,12-dimethylbenz (a) anthraceneinduced experimental oral carcinogenesis. Invest New Drugs 2009; 27: 447-452.

24. Yu Y, Hamza A, Zhang T, Gu M, Zou P, Newman B, Li Y, Gunatilaka $A A$, Zhan CG, Sun D. Withaferin A targets heat shock protein 90 in pancreatic cancer cells. Biochem Pharmacol 2010; 79: 542-551.

25. Sun $C K, N g K T$, Sun BS. The significance of proline-rich tyrosine kinase2 (Pyk2) on hepatocellular carcinoma progression and recurrence. Br J Cancer 2007; 97: 50-57.

26. Sun CK, Man K, Ng KT. Proline-rich tyrosine kinase 2 (Pyk2) promotes proliferation and invasiveness of hepatocellular carcinoma cells through c-Src/ERK activation. Carcinogenesis 2008; 29: 2096-2105.

27. Man K, Lo CM, Xiao JW. The significance of acute phase small for-size graft injury on tumor growth and invasiveness after liver transplantation. Ann Surg 2008; 247: 1049-1057 